\title{
ANÁLISIS DEL COMPORTAMIENTO BIOMECÁNICO DE LA EPÍFISIS FEMORAL EN NIÑOS DE 6 AÑOS AFECTADA POR NECROSIS AVASCULAR: MODELO COMPUTACIONAL \\ ANALYSIS OF THE BIOMECHANICAL BEHAVIOR OF THE FEMORAL EPÍPHYSIS IN CHILDREN OF 6 YEARS AFFECTED BY AVASCULAR NECROSIS: COMPUTATIONAL MODEL
}

\author{
Oscar Rodrigo López V.*, Andrés Fabián Mejía Rojas* \\ * Universidad Santo Tomás, Facultad de Ingeniería Mecánica, GEAMEC \\ Carrera 9 \# 51-11, Bogotá, Cundinamarca, Colombia. \\ E-mail: oscarlopez@usantotomas.edu.co, andresmejiar@usantotomas.edu.co
}

\begin{abstract}
Resumen: La Osteonecrosis (ON) o Necrosis Avascular es una patología caracterizada por ser una enfermedad de debilitamiento de la epífisis de los huesos largos, siendo provocada por la irrupción de flujo sanguíneo. En este trabajo se realiza un análisis biomecánico que permite entender los cambios en las líneas de transferencia de los esfuerzos presentes en la epífisis femoral en presencia de ON en niños de 6 años de edad, y que conducen a la fractura del hueso subcondral, a partir del tipo de lesión necrótica. El análisis de la variación en el comportamiento mecánico se realiza mediante el de método de los elementos finitos, y los dominios de trabajo se construyeron a partir del uso de tomografías computacionales (CT).
\end{abstract}

Palabras clave: Osteonecrosis Avascular, elementos finitos, análisis biomecánico, tomografías computacionales.

\begin{abstract}
Osteonecrosis (ON) or Avascular Necrosis is a pathology characterized by a weakening of the epiphysis of the long bones, caused by the irruption of blood flow. In this work, a biomechanical analysis is carried out to understand the changes in the lines of transfer of the forces present in the femoral epiphysis in the presence of $\mathrm{ON}$ in children of 6 years of age, and that lead to fracture of the subchondral bone, from of the type of necrotic lesion. The analysis of the variation in mechanical behavior is done by the finite element method, and the work domains were constructed from the use of computational tomography (CT).
\end{abstract}

Keywords: Avascular osteonecrosis, finite elements, biomechanical analysis, computed tomography.

\section{INTRODUCCIÓN}

Las alteraciones del flujo sanguíneo en el fémur proximal son causa frecuente de severas deformidades y discapacidades subsecuentes 
(Carrillo 2016). Algunos estudios han demostrado la compleja anatomía vascular de la cadera en desarrollo (Zlotorowicz and Czubak 2014).

En el niño, la cabeza femoral $(\mathrm{CF})$ recibe irrigación sanguínea de vasos epifisarios y vasos que cruzan la región fisaria (Figura 1). Estos vasos desaparecen a medida que la osificación progresa en la CF (Muñoz Carrillo 2009). El patrón de distribución sanguíneo es variable, y cualquier deficiencia puede contribuir al desarrollo de necrosis avascular también conocida como Osteonecrosis $(\mathrm{ON})$.

La ON es una enfermedad producida por la falta temporal o permanente de irrigación sanguínea al hueso, que da lugar progresivamente a la muerte del tejido óseo y posteriormente a la destrucción del hueso (Valle, Noa, and Mas 2010). En correspondencia con trastornos de crecimiento endocondral y osteocondrosis, la ON se presenta como la primera etapa de estas; el ejemplo más evidente es la enfermedad de Legg-Calvé-Perthes (LCP), la cual es una patología pediátrica caracterizada por la necrosis avascular en la $\mathrm{CF}$.

LCP inicia con un período de isquemia que causará necrosis de la CF inmadura, ésta lleva a su fragmentación y a fracturas subcondrales por estrés; con lo que inicia su período clínico y posteriormente pasar a un período de absorción ósea, reosificación y remodelación de la $\mathrm{CF}$, dejando como secuelas alteraciones de la longitud de la extremidad, subluxación o deformidades tanto de la CF como del acetábulo (De Revisión and Austria 2009).

Como resultado de estos eventos isquémicos el crecimiento del centro secundario de osificación, se altera y el hueso se vuelve denso (necrótico). Este hueso denso es luego reabsorbido y reemplazado por hueso nuevo(Hailer, Haag, and Nilsson 2016). Mientras que sucede este proceso las propiedades mecánicas del hueso se alteran y la cabeza femoral tiende a aplanarse y agrandarse (Martínez Lozano 2003).

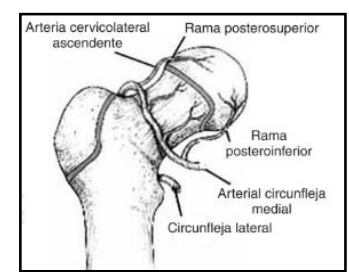

Figura 1. Vascularidad del fémur proximal en desarrollo.
La cabeza femoral se osifica entre el segundo y el octavo meses después del nacimiento y se fusiona con el cuello entre los 15 y 21 años en los niños y un año más temprano en las niñas (Figura 2).

Durante la niñez persiste un puente cartilaginoso entre la epífisis femoral proximal y la apófisis trocantérica a lo largo de la parte superior del cuello femoral. Por esta razón, las fracturas de cadera que cruzan esta área vulnerable pueden producir alteraciones en el crecimiento de la metáfisis, cuello y cabeza femorales.

Existen numerosos métodos de clasificación y valoración respecto al diagnóstico de la $\mathrm{ON}$, sin embargo, resulta complejo realizar un paralelo entre ellas. A través del tiempo, se han desarrollado sistemas de clasificación como auxilio médico que permiten caracterizar la enfermedad.

Entre las distribuciones más aludidas en el campo médico se encuentran, la clasificación de Catterall, la elaborada por Salter y Thompson, y finalmente aquella hecha por Herring (Alvarez and García 2005).

Hoy en día se cuentan con numerosos tratamientos farmacológicos, terapéuticos y quirúrgicos para el cuidado y recuperación de la ON, donde la alternativa a utilizar depende primordialmente del estadio de clasificación en el que se encuentra el paciente (Gómez, Carrillo, and Archila 2010).

Dentro de los tratamientos farmacológicos se encuentra el uso de medicamentos antihipertensivos, antiinflamatorios, y agentes lípidos y anticoagulantes. Estos son usados para aliviar el dolor e inflamación, disminuir los niveles de grasa en la sangre e impedir que los coágulos obstruyan el flujo sanguíneo a los huesos (Valle, Noa, and Mas 2010).

Por otro lado existen tratamientos terapéuticos y de estimulación articular que ejercitan la junta afectada con la finalidad de incrementar la motricidad la misma, sin embargo, estos tratamientos desconocen la fisiopatología de la enfermedad, ya que son utilizados los estadios más prematuros de la enfermedad (López et al. 2014) .

Tras grandes periodos de evolución de la patología, la mayoría de pacientes que padecen ON necesitarán intervención quirúrgica. Bajo esta particularidad, la medicina cuenta con procesos tales como la cirugía de descompresión del núcleo, 
la osteomía, el injerto de hueso y el reemplazo total de la articulación (Martínez-Ferrer, Peris, and Guañabens 2007).

La ley de Wolff, pilar fundamental en el cual se configura este trabajo, establece que la estructura y función del hueso son totalmente independientes, por ello, la ruptura del tejido trabecular en la zona necrótica devasta las líneas de trasferencia de esfuerzo generando cambios en el comportamiento biomecánico y consecuentemente fallas en el apoyo mecánico en junta de la cadera (López et al. 2014), por lo tanto, el objetivo de este trabajo se centra en determinar el cambio en las líneas de trasferencia de esfuerzo por la presencia de $\mathrm{ON}$ en la epífisis femoral en niños de 6 años.

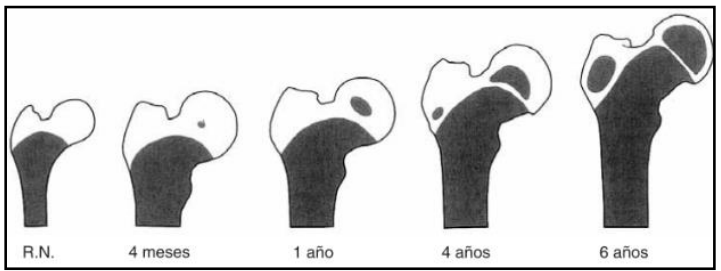

Figura 2. Desarrollo óseo del fémur proximal desde el nacimiento a los 6 años.

\section{JUSTIFICACIÓN}

Los recientes avances en la tecnología, aumentan diariamente el nivel de calidad de vida de las personas; y motivan la aplicación de una medicina más eficiente y menos invasiva (Rodríguez et al. 2007).

Estos avances se deben a la acción interdisciplinaria de investigadores que conjugan conocimientos médicos e ingenieriles. En particular, los desarrollos en la rama de la biomecánica resultan un aporte importante a la ortopedia y traumatología (Panisello, Dupla, and Rodriguez 2000).

Con el rápido desarrollo de la tecnología computacional, se ha implementado vertiginosamente el uso de imágenes médicas en el campo de la salud (Alvarez and García 2012). Con dichas imágenes es posible observar diversas partes del cuerpo humano con el fin de ayudar a los médicos y especialistas a diagnosticar alguna afección que posea el paciente (Mogollon 2000). Además, permiten cuantificar en uno u otro grado enfermedades específicas, como la osteonecrosis avascular, sin la necesidad de someter al paciente a métodos como la cirugía (Schileo et al. 2007).
Por consiguiente, el análisis de elementos finitos (Keshavarzi 2005) se define para este caso como una herramienta muy poderosa para obtener evaluaciones más sofisticadas de la resistencia ósea y el riesgo de fractura relacionado(Schileo et al. 2007).

El creciente número de personas en condiciones pediátricas, que son las más propensas a sufrir de LCP, el elevado costo de la experimentación invitro, el desconocimiento del comportamiento anatómico del fémur bajo diferentes estadios de la enfermedad, entre otros factores, han motivado un mayor protagonismo de la biomecánica computacional, marco en el cual se desarrolla este trabajo.

A nivel nacional se han encontrado pocos trabajos de investigación sobre la ON (Mogollon 2000). Si se hace énfasis en lo anterior es evidente la necesidad de realizar más estudios con el objetivo de caracterizar, fortalecer y sobre todo comprender el curso natural de la enfermedad y, así sea posible aportar elementos de juicio que permitan validar los métodos actuales para el tratamiento de la afección (Simanca 2018).

Por ello, determinar el comportamiento biomecánico de la epífisis bajo un escenario necrótico permitirá obtener información de la variación del estímulo mecánico sobre el tejido cartilaginoso y óseo, insumo fundamental para la realización de modelos mecanobiológicos o fenomenológicos que permitan estudiar los procesos de modelado, remodelado y daño en el crecimiento endocondral.

\section{MÉTODOS}

Se reconstruye la cadera de una niña de 6 años de edad a partir de tomografías computacionales. Las lesiones producidas por la $\mathrm{ON}$ de acuerdo al estadio y porcentaje de afectación en el volumen de la epífisis femoral fueron elaboradas haciendo énfasis en la clasificación establecida por Herring.

Se elaboró un modelo bajo condiciones sanas y un modelo patológico. El modelo sano se utilizó con el fin de determinar y validar el comportamiento biomecánico de la epífisis femoral sin ninguna contribución patológica (Figura 3). Para ambos modelos se diseñan los cartílagos de crecimiento y, se reconocen las diferentes densidades óseas que constituyen el hueso (hueso trabecular y cortical). Asimismo, se elabora el respectivo diseño y reconstrucción ósea que corresponde a los tejidos 
necrosados como se muestra en la como se muestra en la figura 4.

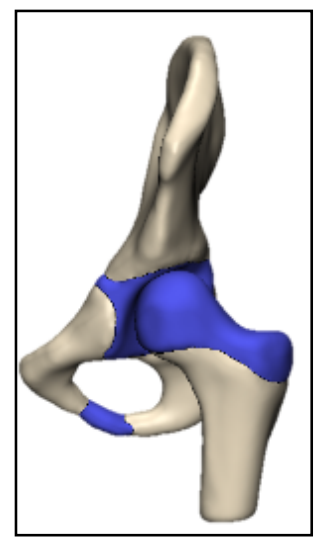

Figura 3. Reconstrucción ósea de cadera sana niña de 6 años.

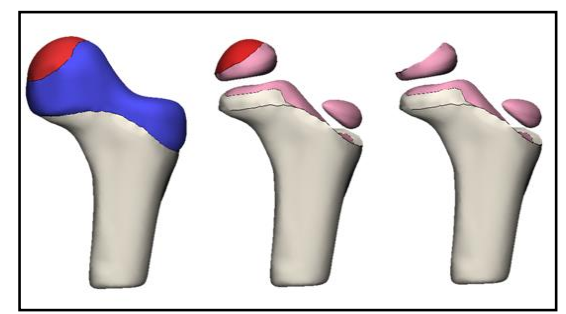

Figura 4. Reconstrucción ósea del fémur con necrosis avascular tipo $C$.

Las propiedades mecánicas para los tejidos óseos y los cartílagos se obtuvieron de los estudios técnicos reportados y validados en la literatura especializada (Alvarado, Roa, and Cortés 2004)(Kluess et al. 2010)(Schileo et al. 2007) (Tabla 1). La asignación de propiedades mecánicas para cada uno de los tejidos fue realizada asumiéndolos como un material bilineal, es decir apreciando su propiedad de plasticidad y una estructura anisotropíca del fémur para así determinar el cambio en las líneas de trasferencia de esfuerzo.

Se establecen las condiciones de frontera de acuerdo a las condiciones de carga producidas en la cadera para una posición bipodal erguida y producidas por los músculos de los glúteos, ligamento iliofemoral y peso del paciente. Las cargas se definieron de acuerdo al modelo presentado por Pauwells y Brinckmann (Tönnis).

De acuerdo a esto, como primer condición de carga se aplica una presión sobre el cartílago de la epífisis femoral en un área equivalente a la zona donde se encuentra el ligamento iliofemoral, por otra parte, en la superficie de la cresta iliaca y bajo un área semejante a la línea de acción muscular del glúteo mayor, se ubica la segunda condición.

Las opciones de contacto fueron definidas entre las superficies de los diferentes tejidos interconectados (hueso cortical - hueso trabecular, hueso cortical cartílago y hueso trabecular - lesión), se tomaron contactos tipo Bonded debido a que este permite la unión de las superficies en todas las direcciones evitando deslizamientos y la separación entre ellas, de igual forma como ocurre en la realidad entre las superficies interconectadas de los tejidos.

Tabla 1. Propiedades mecánicas de los elementos mecánicos

\begin{tabular}{|c|c|c|c|}
\hline Componente & $\begin{array}{c}\text { Modulo } \\
\text { Elástico } \\
(\text { Mpa })\end{array}$ & $\begin{array}{l}\text { Coeficiente } \\
\text { de Poisson }\end{array}$ & Autor \\
\hline $\begin{array}{c}\text { Hueso } \\
\text { Cortical }\end{array}$ & 17000 & 0,3 & $\begin{array}{c}\text { (Sunghyen \& } \\
\text { Mistugu, 2013) }\end{array}$ \\
\hline $\begin{array}{c}\text { Hueso } \\
\text { Trabecular }\end{array}$ & 1500 & 0,33 & $\begin{array}{c}\text { (Voo, Armand, } \\
\text { \& kleinberger, } \\
\text { 2004) }\end{array}$ \\
\hline $\begin{array}{l}\text { Cartílago } \\
\text { acetabular }\end{array}$ & 1,236 & 0,043 & $\begin{array}{c}\text { (Gozzoli Pietro, } \\
\text { 2017) }\end{array}$ \\
\hline $\begin{array}{l}\text { Cartílago } \\
\text { epifisiario }\end{array}$ & 1,186 & 0,039 & $\begin{array}{c}\text { (Gozzoli Pietro, } \\
\text { 2017) }\end{array}$ \\
\hline $\begin{array}{l}\text { Cartílago } \\
\text { necrosado }\end{array}$ & 0,8 & 0,35 & $\begin{array}{c}\text { Valores } \\
\text { establecidos } \\
\text { por el autor } \\
\text { bajo carácter } \\
\text { experimental }\end{array}$ \\
\hline $\begin{array}{l}\text { Osificación } \\
\text { lesionada }\end{array}$ & 100 & 0,4 & $\begin{array}{l}\text { Valores } \\
\text { establecidos } \\
\text { por el autor } \\
\text { bajo carácter } \\
\text { experimental }\end{array}$ \\
\hline
\end{tabular}

La malla de elementos finitos para ambos modelos se generó mediante el uso de métodos adaptativos para geometrías orgánicas como se ve en la (Figura 5). Se emplearon elementos tetraédricos 3D y se realizó un análisis de convergencia para validar la aproximación del método numérico, de tal forma que se obtuvo un enmallado de 162.280 elementos con 261.146 nodos 


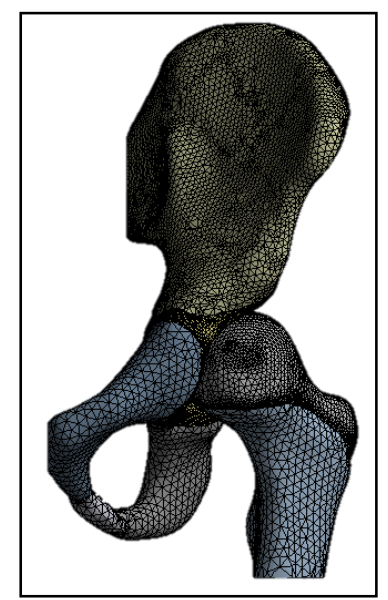

Figura 5. Malla de elementos finitos generada para las geometrías orgánicas.

\section{CONCLUSIONES}

Con el fin de validar los modelos computacionales, inicialmente se realizó una la simulación de los elementos de trabajo suponiendo condiciones óptimas para cada uno de los tejidos, es decir, dentro de una atmosfera normal y sin ninguna patología; posteriormente, se evaluaron los resultados para el modelo en escenario necrótico. Ambos modelos fueron comparados de acuerdo a los desplazamientos que la cadera sufre, la distribución de esfuerzos presentes en la epífisis femoral, la distribución de esfuerzos en el cartílago acetabular y finalmente los esfuerzos presentes en el centro secundario de osificación.

A pesar de que la necrosis avascular es una enfermedad con una complejidad alta en su curso natural, a la fecha no están completamente comprendidos los mecanismos que conllevan a su inicio. Aunque se han desarrollado varios estudios alrededor de esta patología, estos se han enfocado a la determinación de los factores que conducen a los inicios de la enfermedad basados en análisis radiológicos (análisis descriptivos) y biológicos (Anal and Araujo 2007). De esta forma Harry K.W. et al. (Kim 2011) determinaron a través de estudios experimentales indicios que los factores mecánicos y biológicos contribuyen al desarrollo de la cabeza femoral. De acuerdo a esto, son escasos los estudios que permitan establecer la importancia del estímulo mecánico en el deterioro, modelado o remodelado de la epífisis bajo un escenario necrótico. La experimentación computacional ha surgido como una herramienta que permite mejorar el entendimiento sobre el desarrollo biológico de patologías complejas, de esta forma los modelos biomecánicos que se desarrollan en relación a dichas afecciones médicas, surgen como una alternativa para el entendimiento de la biología básica del proceso del modelado y regeneramiento óseo, disminuyendo tiempos y evitando experimentación en seres vivos.

Aunque no existen reportes documentados de como varían las propiedades mecánicas de los tejidos afectados por la necrosis, en este trabajo se asumió una disminución en la rigidez de los tejidos involucrados (cartílago epifisial y centro primario de osificación), por lo que en el centro secundario de osificación el módulo de rigidez se disminuyó de 400 a $100 \mathrm{MPa}$ lo que indica una reducción del $75 \%$ y en el cartílago epifisial se disminuyó de 1,186 a $0,8 \mathrm{MPa}$ representando una reducción del $32 \%$. Como resultado de esta variación en las propiedades mecánicas se puede observar que en el modelo afectado por necrosis los valores de esfuerzo aumentaron en un $65 \%$ dentro de la epífisis femoral. Por otro lado, en el modelo sano se puede apreciar que las condiciones de carga, y la transferencia de esfuerzos en la articulación favorece el estímulo mecánico en la placa de crecimiento (Figura 6a) y así mismo se evidencia una reciprocidad en el estímulo mecánico que se produce en el hueso trabecular presente en el centro secundario de osificación y en hueso trabecular del íleon, condiciones que favorecen los procesos biológicos para el remodelado y modelado del tejido óseo. Comparando estás condiciones en el modelo afectado por necrosis se evidencia que por la presencia de esta patología el estímulo mecánico en la placa de crecimiento (fundamental para el proceso de osificación endocondral) se disminuye en un $42 \%$ y adicionalmente el centro secundario de osificación aumenta sus esfuerzos en su zona media (zona colindante entre el hueso trabecular sano y el necrosado) en un $37 \%$, lo que indica la posible presencia de fracturas trabeculares producto de los sobreesfuerzos presentes.

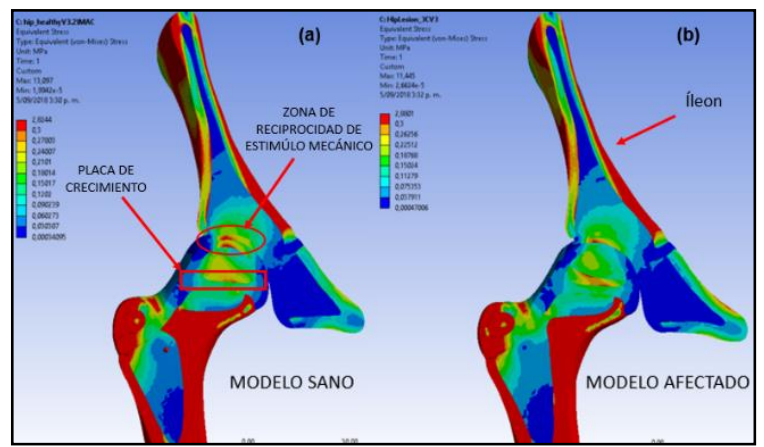

Figura 6. Comparación de resultados entre los modelos computacionales. 
Las diferencias entre el modelo sano y el modelo afectado con necrosis avascular, inicialmente radican en la distribución y magnitud de los esfuerzos dentro de la cabeza femoral. Esto evidentemente es producto de la disminución de la rigidez en los tejidos necrosados, lo que conlleva a un cambio en la distribución de las líneas de isoesfuerzo según lo planteado en la ley de Wolff, por lo que el esfuerzo en el modelo patológico se concentra en la zona media del centro secundario de osificación (Figura $6 \mathrm{~b}$ ) lo que puede propiciar el inicio de fracturas o retrasos en la osificación endocondral por las nuevas condiciones de esfuerzos.

Debido a la transferencia de esfuerzos presentes en la articulación coxofemoral sana, se genera un estímulo mecánico en la zona de la placa de crecimiento que posteriormente da lugar a la osificación del cartílago epifisiario (Figura 6a). Por otro lado, los esfuerzos que se hallan en la zona de reciprocidad del cartílago acetabular y el cartílago epifisial, se traducen como un estímulo mecánico para el remodelado óseo entre el hueso trabecular del ilion y el hueso trabecular del fémur.

Dadas las condiciones patológicas para el modelo afectado y lo que se aprecia en la figura $6 \mathrm{~b}$, la transferencia de cargas por parte del cartílago acetabular hacia la epífisis femoral es interrumpida debido a la falta de reciprocidad mecánica entre los cuerpos de contacto, causando una posible distorsión de la cabeza femoral. El centro secundario de osificación al estar bajo escenario necrótico muestra una sobrecarga de esfuerzos en su zona media, inhibiendo la transmisión de esfuerzos hacia la placa de crecimiento, lo que posiblemente implica un retraso al proceso de osificación endocondral, y posteriormente dar lugar a la fractura subcondral.

A pesar de todas estas diferencias la distribución de esfuerzos comparado con lo encontrado en la literatura especializada es similar y, el rango de los esfuerzos está dentro de la misma magnitud. Esto permite concluir que el trabajo realizado a pesar de sus simplificaciones permite establecer un comportamiento biomecánico que no se aleja de la realidad biológica y permite el entendimiento de patologías relacionados con el malfuncionamiento de diferentes tejidos presentes en la articulación coxofemoral.

\section{RECONOCIMIENTO}

Este trabajo se realizó con recursos de la decimosegunda convocatoria interna para el fomento de la investigación FODEIN-2018 de la Universidad Santo Tomás.

\section{REFERENCIAS}

Alvarado, Diego, Máximo Roa, and Carlos Cortés. 2004. Analisis Por Elementos Finitos Del Proceso de Regeneración Ósea.

Alvarez, Alejandro, and Yenima García. 2005. "Clasificación y Diagnóstico de La Osteonecrosis de Cabeza Femoral." Medicina, 58-65.

Alvarez, Alejandro, and Yenimia García. 2012. "Clasificación y Diagnóstico de La Osteonecrosis de Cabeza Femoral." Perspectives in Science 1 (1).

Anal, Cecilia, and Ezequiel Mateo Araujo. 2007. "Sindrome de Legg Calve Perthes." Revista de Posgrado de La VIa Cátedra de Medicina. 1: 10-14.

Bonfante, M., \& Castillo, A. (2014). Integración de sistema multi-agente, ontologías y procesos de negocios como marco tecnológico de la estrategia "gobierno en línea". REVISTA COLOMBIANA DE TECNOLOGÍAS DE AVANZADA, 1(23).

Carrillo, Hugo. 2016. "Fracturas de Cadera En El Niño." Medigraphic Artemisa 5 (3): 1-7. http://www.saludalia.com/saludfamiliar/fracturas-de-cadera-en-el-anciano.

C. Ferrin, X. Magdalena, H. Loaiza, S. López, S. Henao. (2014). Sistema de extracción automática de parámetros morfológicos de la huella plantar mediante técnicas de visión por computador en un sistema embebido. REVISTA COLOMBIANA DE TECNOLOGÍAS DE AVANZADA, 1(23).

Ferrin, C., Magdalena, X., Loaiza, H., López, S., \& Henao, S. (2014). Sistema de extracción automática de parámetros morfológicos de la huella plantar mediante técnicas de visión por computador en un sistema embebido. REVISTA COLOMBIANA DE TECNOLOGÍAS DE AVANZADA, 1(23). 
Gómez, Andrés, Mauricio Carrillo, and Faber Archila. 2010. "Estudio de Ayudas Tecnicas Tipo Exoesqueleto Para Apoyo a Pacientes Con Desordenes En El Movimiento." Revista Colombiana de Tecnologías de Avanzada, 52-59.

Hailer, Yasmin D, Anna C Haag, and Olof Nilsson. 2016. "Legg-Calvé-Perthes Disease: Quality of Life, Physical Activity, and Behavior Pattern." Journal of Pediatric Orthopedics 34 (5): 514-21.

Keshavarzi, Naghmeh. 2005. "Un Modelo Computacional Para Flujo Poroso En Sistemas Subterraneos." Revista Colombiana de Tecnologías de Avanzada, 74-80.

Kim, Harry K W. 2011. "Legg-Calve-Perthes Disease: Etiology, Pathogenesis, and Biology." LCPD Supplement 31 (2): 141-46.

Kluess, Daniel, Jan Wieding, Robert Souffrant, Wolfram Mittelmeier, and Rainer Bader. 2010. "Finite Element Analysis in Orthopaedic Biomechanics." Finite Element Analysis 9 (4): 151-71.

L. A. M. Mesa, N. B. Lombana. (2013). LA ROBÓTICA EDUCATIVA COMO INSTRUMENTO DIDÁCTICO ALTERNATIVO EN EDUCACIÓN BÁSICA. REVISTA COLOMBIANA DE TECNOLOGÍAS DE AVANZADA, ISSN: 1692-7257. 2( 22).

L. Mesa Mesa and N. Barrera Lombana. (2013). LA ROBÓTICA EDUCATIVA COMO INSTRUMENTO DIDÁCTICO ALTERNATIVO EN EDUCACIÓN BÁSICA. REVISTA COLOMBIANA DE TECNOLOGÍAS DE AVANZADA, vol. 2, no. 22 .

López, Rodrigo, Juan Ruiz, Leonardo Pineda, and Universidad Santo Tomás. 2014. "Comportamiento Biomecánico de La Epífisis Femoral En Presencia de Osteonecrosis."

M. C. Bonfante, A. Castillo. (2014). Integración de sistema multi-agente, ontologías y procesos de negocios como marco tecnológico de la estrategia "gobierno en línea". REVISTA COLOMBIANA DE TECNOLOGÍAS DE AVANZADA, ISSN:
1692-7257. 1(23).

Martínez-Ferrer, M. A., Pilar Peris, and Nuria Guañabens. 2007. "Osteonecrosis. ¿Qué Hay de Nuevo?” Reumatologia Clinica 3 (2): 78 84.

Martínez Lozano, Aurelio G. 2003. "Enfermedad de Legg-Calvé-Perthes. Conceptos Actuales." Revista Mexicana de Ortopedia Pediátrica 5 (1): 5-11.

Mogollon, Maria. 2000. "Incidencia de Necrosis Avascular de Cabeza de Femur En Pacientes Adultos." Universidad Centroccidental "Lisandro Alvarado."

Muñoz Carrillo, Hugo. 2009. "Fracturas de Cadera En El Niño." Medicina 5 (3): 1-7. http://www.saludalia.com/saludfamiliar/fracturas-de-cadera-en-el-anciano.

Panisello, Sebastian, Lallana Dupla, and Herrera Rodriguez. 2000. "Necrosis de La Cabeza Femoral Tras Fractura Del Cuello Femoral Tratada Mediante Osteosíntesis" 35 (201): 11-13.

Revisión, Artículo De, and Raúl Frías Austria. 2009. "Enfermedad de Legg-Calvé-Perthes." Acta Ortopédica Mexicana 23 (3): 172-81.

Rodríguez, Lelis, Miguel Ocampo, Abúndez Pliego, Navarro Torres, and Piña Piña. 2007. "Estudio de Transmisión de Carga En El Fémur Humano." Ingeniería Mecánica Tecnología y Desarrollo 2 (5): 164-68.

Schileo, Enrico, Fulvia Taddei, Andrea Malandrino, Luca Cristofolini, and Marco Viceconti. 2007. "Subject-Specific Finite Element Models Can Accurately Predict Strain Levels in Long Bones." Journal of Biomechanics 40 (13): 2982-89.

Simanca, Pedro León. 2018. Innovación Para Un Nuevo País.

Valle, Maikel, Miriam Noa, and Rosa Mas. 2010. "Osteonecrosis. Una Breve Revisión." CENIC Ciencias Biológicas 41 (2): 91-97.

Zlotorowicz, Marcin, and Jaroslaw Czubak. 2014. "Vascular Anatomy and Blood Supply to the Femoral Head" 2 (1): 19-26. 